\title{
Article \\ Functionalized Mesoporous Silica Nanoparticles as Delivery Systems for Doxorubicin: Drug Loading and Release
}

\author{
Candace M. Day ${ }^{1}\left(\mathbb{D}\right.$, Martin J. Sweetman ${ }^{1,2} \mathbb{D}$, Yunmei Song ${ }^{1}$, Sally E. Plush ${ }^{1,2, *}$ and Sanjay Garg ${ }^{1,2, *(\mathbb{D})}$ \\ 1 Clinical and Health Sciences, University of South Australia, North Terrace, Adelaide, SA 5000, Australia; \\ Candace.Day@mymail.unisa.edu.au (C.M.D.); Martin.Sweetman@unisa.edu.au (M.J.S.); \\ May.Song@unisa.edu.au (Y.S.) \\ 2 Future Industry Institute, University of South Australia, Mawson Lakes, SA 5095, Australia \\ * Correspondence: Sally.Plush@unisa.edu.au (S.E.P.); sanjay.Garg@unisa.edu.au (S.G.); \\ Tel.: +61-8-8302-2586 (S.E.P.); +61-8-8302-1575 (S.G.)
}

check for updates

Citation: Day, C.M.; Sweetman, M.J.; Song, Y.; Plush, S.E.; Garg, S.

Functionalized Mesoporous Silica Nanoparticles as Delivery Systems for Doxorubicin: Drug Loading and Release. Appl. Sci. 2021, 11, 6121. https://doi.org/10.3390/app11136121

Academic Editor: Greta Varchi

Received: 27 May 2021

Accepted: 28 June 2021

Published: 30 June 2021

Publisher's Note: MDPI stays neutral with regard to jurisdictional claims in published maps and institutional affiliations.

Copyright: (c) 2021 by the authors. Licensee MDPI, Basel, Switzerland. This article is an open access article distributed under the terms and conditions of the Creative Commons Attribution (CC BY) license (https:// creativecommons.org/licenses/by/ $4.0 /)$.

\begin{abstract}
Functionalized nanoparticles have played a major role in the field of targeted therapy, owing to their ability to control the release and for the selective delivery of entrapped materials to tumours. In this work, we described the loading capacity and in vitro release kinetics of mesoporous silica nanoparticles (MSNs), functionalized with Poly-L-Histidine and Tamoxifen. The model drug Doxorubicin (DOX) was successfully encapsulated into MSN-based systems, using the technique of solvent immersion. A post-surface grafting loading method was investigated on functionalized systems, with DOX loading content determined using HPLC. Dialysis bag diffusion was employed to investigate the release kinetics of DOX-loaded-systems at $\mathrm{pH} 7.4$ and 5. The amount of DOX released from native MSNs systems over a $72 \mathrm{~h}$ period at $\mathrm{pH} 5$ was approximately $40 \%$; and at $\mathrm{pH} 7.4 \approx 30 \%$. A moderate $\mathrm{pH}$ dependent release behaviour was observed with both our functionalized systems: DOX@MSN-PLH and DOX@MSN-PLH-TAM; with approximately 5\% of DOX released from DOX@MSN-PLH-TAM at pH 7.4 and about $9 \%$ released at pH 7.4 over $72 \mathrm{~h}$. The maximal cumulated release of DOX molecules from DOX@MSN-PLH after $72 \mathrm{~h}$ was $\approx 18 \%$ at $\mathrm{pH} 7.4$ and $\approx 23 \%$ at $\mathrm{pH} 5$, respectively. The outcome of this work offers a promising contribution towards building future stimuli-responsive nano-drug delivery systems.
\end{abstract}

Keywords: mesoporous silica nanoparticles; doxorubicin; tamoxifen; Poly-L-Histidine; drug delivery

\section{Introduction}

The recent trends of using nanotechnology to resolve unmet medical needs have been recognized as a Key Enabling Technology in the European Union (EU) [1]. By developing advanced drug delivery systems (DDSs), therapeutic compounds of interest can be entrapped within nanoparticle matrices or conjugated to different nanosystems [2,3], drug molecules can be delivered to designated molecular targets at much lower doses, and over longer time periods [2-4]. The development of nanoformulations has revolutionized the delivery and targeting of therapeutic compounds, providing an economic and innovative utilization of existing drugs [5-9].

Numerous nanocomposites have received considerable attention as promising DDSs. Mesoporous silica nanoparticles (MSNs), discovered in 1992 by the Mobile Oil Corporation, have emerged as one of the most advanced and versatile nano DDSs [10-13]. Small sized MCM-41 MSNs are the most frequently used carriers for pharmaceutical applications, owing to their unique mesoporous structure, high surface area, large pore volume and tuneable pore diameter [10-13]. In addition to this, MSNs also provide a solid framework with abundant surface chemical moieties, allowing surface functionalization with various ligand molecules, for the targeted and controlled release of encapsulated drug moieties to a particular site $[10,11,14-16]$. 
Even though MSNs has been widely used in drug delivery, considerate efforts towards novel compositions of MSN-based systems have been attempted through surface engineering with different targeting functionalities, to enhance the accuracy and selectivity of these systems as nanocarriers, beyond that of unfunctionalized MSNs. As a result, recently we presented an exciting conceptual study in which a novel functionalized MSNbased system was synthesized and characterized (Figure 1a) [16]. The fabricated MSNs are highly uniformed in spherical shapes and sizes $(\approx 150 \mathrm{~nm})$, with well-ordered porous channels, without the interconnection between each individual channel (Figure 1b). This represented the first step of our journey into the development of a nano modality with the ability to transport anticancer agents selectively to breast tumours, to reduce off-target adverse effects. In that work, the $\mathrm{pH}$-sensitive polymer Poly-L-Histidine (PLH) and the FDA-approved hormonal drug Tamoxifen (TAM) were appended to native MSNs. Our functional nanostructure is believed to lay a foundation for a new theoretical concept of triple-action DDSs, which includes: (i) passive targeting using nanosized MCM-41 as a DDS; (ii) active targeting through the selective binding of TAM to estrogen receptors (ERs)-overexpressing-breast tumours; (iii) controlled drug release with PLH molecules acting as a $\mathrm{pH}$ sensitive gatekeeper, preventing premature drug leakage [16].

(a)

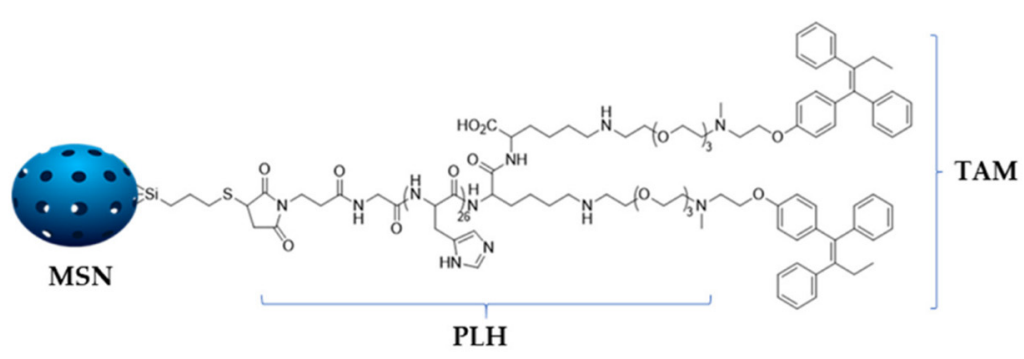

(b)

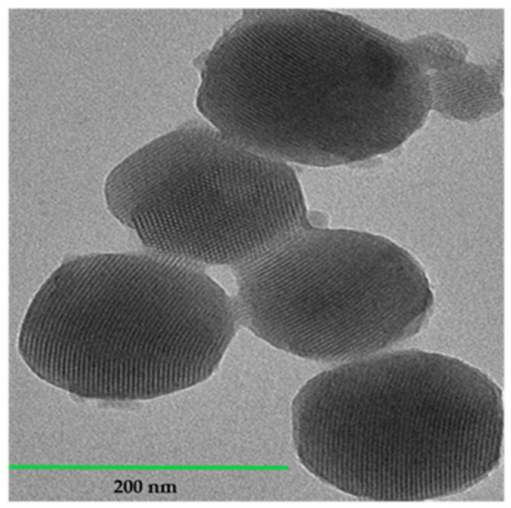

Figure 1. (a) The triple-action nanostructure DDS based on MSNs, functionalized with the pH sensitive PLH polymer and the hormonal drug Tamoxifen. Image adapted from Day et al. [16]. (b) TEM images demonstrating the diameter and internal structure of MSNs on the $200 \mathrm{~nm}$ scale-bar, demonstrating well-defined ordered long-range structure of inner mesopores and highly ordered porous channels.

A high drug loading capacity (the mass ratio of drugs to nanosystems) is an important parameter to determine whether a nanostructure can be employed as an ideal DDS [17]. In this study, we will load our previously synthesized functionalized nanostructures with Doxorubicin (DOX), to investigate loading ability of our system. DOX has been proven to be effective against both early and metastatic breast cancer, owing to its ability to interfere with DNA and RNA synthesis in solid tumours [18-20]. However, the potential clinical applications of DOX are hindered by its highly toxic profile and non-specific mechanisms of action which can cause fatal damage to healthy and sensitive tissues, leading to cardiac arrest in some cases $[19,20]$. Therefore, successful loading of DOX onto MSN-based systems 
is expected to enhance its delivery, via both passive diffusion and active targeting with functionalized MSNs.

There are two general methods by which drug molecules such as DOX are loaded onto functionalized MSNs systems: (i) pre-surface grafting [21-27] (Figure 2a) and (ii) postsurface grafting (Figure 2 b) which is less frequently applied $[28,29]$. For the pre-surface grafting method as illustrated in Figure 2a, native MSNs are immersed into a stirred solution of DOX, the DOX molecules then diffuse into the porous channels of the MSNs. The resulting DOX@MSNs (in which @ means loaded) are then grafted with PLH and TAM, forming DOX@MSN-PLH-TAM (Figure 2a). For the post-surface grafting method, fully functionalized MSN-PLH-TAM will be immersed into a stirred solution of DOX (Figure $2 b$ ). The resulting DOX@MSN-PLH-TAM particles are then collected by centrifugation. In this study, we are particularly interested in the loading of DOX into our novel MSN-based systems (Figure 1), using the unconventional post-surface grafting loading approach (Figure $2 \mathrm{~b}$ ). This is expected to avoid the leakage of DOX molecules which may occur during each step of surface functionalization with the pre-surface grafting method.

(a)

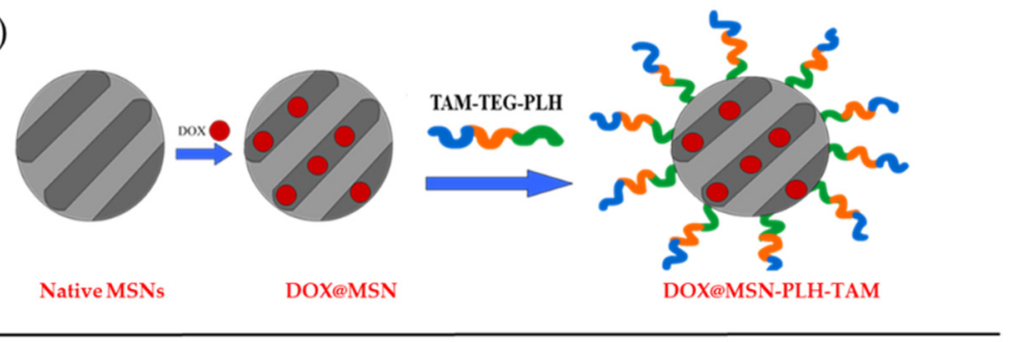

(b)

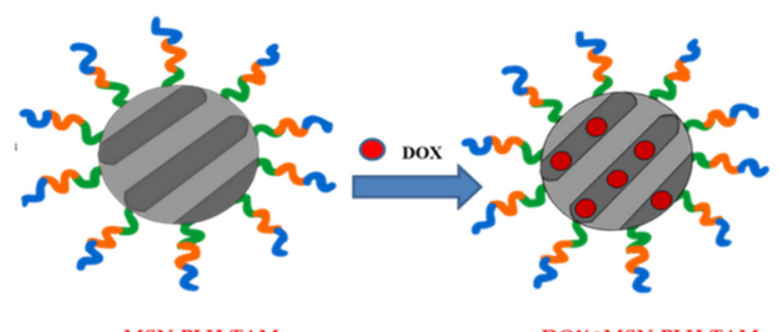

MSN-PLH-TAM

DOX@MSN-PLH-TAM

Figure 2. Illustration of the pre-grafting loading of MSN-based system. (a) MSNs were firstly loaded with DOX molecules, followed by surface functionalization to yield DOX@MSN-PLH-TAM in preloading grafting methods. (b) Fully functionalized MSN-PLH-TAM systems are loaded which DOX to form DOX@MSN-PLH-TAM in post-grafting-loading method.

Additionally, in this study, we focused on the in vitro release kinetics of our functionalized systems, attributed to the grafted PLH molecules. With the theory that PLH molecules which have a $\mathrm{p} K_{\mathrm{a}} \sim 6.10$, under physiological conditions ( $\mathrm{pH}$ 7.4), will condense on the surface of MSNs. This will block the MSN pores and prevent the release of DOX [16]. Subsequently, as the PLH-gated nanosystems enter into tumours, the PLH molecules will become protonated by the acidic tumour environment $(\mathrm{pH} \sim 5)$. The cationic PLH molecules, due to charge repulsion, are then expected to extend out from the surface of the MSNs, thus opening the pores to release DOX [16]. In addition to this, it was also hypothesized that the greater solubility of DOX molecules in acidic environments (pH 5) [30,31] in combination with the introduction of PLH molecules onto MSNs should enhance the release of entrapped DOX molecules in acidic tumour microenvironments.

\section{Materials and Methods}

\subsection{General Methods and Materials}

TAM was obtained from Combi-Blocks Inc. (San Diego, CA, USA). PLH (Mal-PLH$\mathrm{NH}_{2} / \mathrm{Mal}-\mathrm{GH}_{26} \mathrm{KK}$, purity $90 \%, 26$ units, $\mathrm{Mw}=4.05 \mathrm{kDa}$ ) was purchased from Leon 
Biological Technology Co. Ltd. (Nanjing, China). Doxorubicin.HCl was obtained from Sapphires Bioscience Ltd. (Adelaide, Australia). All other reagents were purchased from Sigma Aldrich and used without further purification. All $\mathrm{pH}$ measurements were conducted on a pre-calibrated Orion Ross $\mathrm{pH}$ meter (Metrohm AG, Herisau, Switzerland). RP-HPLC Chromatography was performed on a Shimadzu HPLC system (Kyoto, Japan) equipped with a PDA detector (start $\lambda 200 \mathrm{~nm}$, end $\lambda 350 \mathrm{~nm}$, analysed at $250 \mathrm{~nm}$ ), using a C18 column $(4.6 \times 150 \mathrm{~mm}, 5 \mu \mathrm{m})$ as the stationary phase. The mesostructures and diameters of MSN-PLH-TAM were analysed by high-resolution transmission electron microscopy (HR-TEM) (Hitachi S-4800, Tokyo, Japan) obtained on an HC electron microscope at an accelerating voltage of $100 \mathrm{kV}$.

\subsection{Synthesis of Native MSN, MSN-PLH and MSN-PLH-TAM}

The synthesis of three MSN-based systems; namely native MSNs, MSN-PLH and MSN-PLH-TAM directly followed our recently published study [16]. Native MSNs were synthesized via the Stöber method, using CTAB as the templating structure and TEOS as the silica precursor. The surface (OH-) moieties of MSNs were converted to thiols using MPTES and conjugated to Mal-PLH- $\mathrm{NH}_{2}$ molecules via Michael addition at $\mathrm{pH} 5$, to give MSN-PLH. Resulting MSN-PLH nanostructure was then reacted with mesylated TAM-TEG building blocks prepared in-house (in which TEG- Tetraethylene glycol was used as an organic linker) to give the requisite MSN-PLH-TAM nanomaterial.

\subsection{Loading of DOX onto MSN-Based Systems}

The solvent immersion technique for loading of native MSNs was adapted and modified from a previous study by Hakeem and colleagues [32]. MSNs (5 mg) were ultrasonically dispersed in $5 \mathrm{~mL}$ solution of DOX in MilliQ water (DOX conc $=1 \mathrm{mg} \mathrm{mL}^{-1}$ ) for $15 \mathrm{~min}$. The suspension remained under stirring for $24 \mathrm{~h}$ at room temperature, protected from light. DOX@MSN was collected using centrifugation (12,000 RPM, $\left.30 \mathrm{~min}, 24^{\circ} \mathrm{C}\right)$. The supernatant was collected separately; while solid DOX@MSNs were washed with MilliQ water $(2 \mathrm{~mL} \times 3)$ until supernatant became clear, indicating the absence of free DOX particles in the wash. HPLC was then employed to quantify the amount of free/unloaded DOX molecules in the combined supernatants. The HPLC analytical method was adapted and modified from a previously published procedure [33]. From the free DOX determined by HPLC, loaded DOX can be quantified based on the initial amount of DOX in the original solution. A calibration curve was obtained from a serial dilution of stock solution containing pure DOX in MilliQ water (concentration $1 \mathrm{mg} \mathrm{mL}^{-1}$ ). Encapsulation efficiency $(\mathrm{EE} \%)$ and drug content (DC\%) were determined using the following Equations (1) and (2), respectively [34,35].

$$
\mathrm{EE} \%=\text { Weight of loaded DOX/Initial weight of DOX } \times 100 \%
$$

$\mathrm{DC} \%=$ Amount of loaded DOX $/($ Weight of MSNs + Weight of loaded DOX $) \times 100 \%$

A previous procedure from Bilalis et al. [36] was adapted and modified for the postsurface grafted loading of MSN-PLH and MSN-PLH-TAM (Figure 2b). In short, MSN-PLH or MSN-PLH-TAM (15 mg) were ultrasonically dispersed in $6.25 \mathrm{~mL}$ of PBS buffer ( $\mathrm{pH}$ 5) containing DOX (concentration $1.5 \mathrm{mg} \mathrm{mL}^{-1}$ ). The resulting solutions were then stirred vigorously (400 RPM) at room temperature and protected from light for $24 \mathrm{~h}$. DOX@MSNPLH and DOX@MSN-PLH-TAM were collected by centrifugation (12,000 RPM, 20 min, $\left.24^{\circ} \mathrm{C}\right)$, and washed with water $(2 \mathrm{~mL} \times 3)$. Total supernatant and washing solvents were recovered and HPLC was then employed to quantify EE\% and DC\% of both systems following Equations (1) and (2) above.

\subsection{In-Vitro Release Kinetics}

To investigate the in vitro release pattern of DOX@MSN, DOX@MSN-PLH and DOX@MSN-PLH-TAM, a dialysis diffusion technique was employed. To mimic systemic 
circulation where $\mathrm{pH}$ is 7.4 , and the tumour environment where $\mathrm{pH}$ is $\approx 5$; $\mathrm{PBS}$ buffer solutions at each of the respective $\mathrm{pHs}$ were employed as releasing media. Then, $1 \mathrm{mg}$ of each sample was dispersed with buffer solution $(1 \mathrm{~mL})$ and placed in cellulose membrane dialysis bag, which was then placed in $10 \mathrm{~mL}$ of respective release media at $37 \pm 0.5^{\circ} \mathrm{C}$, with $100 \mathrm{rpm}$ paddle rotation. At 2, 4, 6, 24, 28 and $72 \mathrm{~h}$, aliquots of sample $(1 \mathrm{~mL})$ were withdrawn and replenished with the same volume of fresh media. Pure DOX in buffers was used as positive controls, while the suspensions of native MSNs in buffers were used as negative control.

\section{Results and Discussion}

\subsection{Drug Loading}

For the pre-surface grafting method (Figure 2a), when DOX is loaded into the native MSNs, the colour of particles changed from white to red, indicating the presence of DOX molecules in the sample. According to the data obtained by HPLC, DOX@MSNs achieved an EE of $7.78 \pm 0.18 \%$, and a DC of $7.22 \pm 0.153 \%$. This was in accordance with the original data by which this procedure was adapted, reporting an EE of $\sim 6.5 \%$ by weight [32]. Even though in their original study, Hakeem et al. did not report their DC result; the obtained DC value of $\sim 7 \%$ was also in accordance with other literature procedures which have reported DC values of less than 10\% [36]. No extra signals were observed in the FT-IR of the DOX@MSN following encapsulation (see Figure S1 in Supplementary Material). This suggests that the loaded DOX content might be just too low to be detected by FT-IR.

Following the loading of DOX into the MSNs, the DOX@MSNs was then functionalized with PLH and TAM as per our previous work (see Supplementary Material for full characterization) [16]. The synthesis is a continuous/one-pot reaction, in which the resulting DOX@MSN-PLH was immediately reacted with TAM-TEG-OMs, to yield DOX@MSNPLH-TAM. It was assumed that the maintenance of red colour indicated that the DOX molecules remained inside the porous channels during the grafting steps. This is as per previous literatures $[37,38]$, and no obvious red colour of the washing solutions was observed. Therefore, at the end of surface grafting, DOX@MSN-PLH-TAM was assumed to have an EE of $7.78 \pm 0.18$, similar to that of DOX@MSNs. This assumption was considered during the in vitro release experiments.

The colour change upon DOX loading was also observed for our functionalized MSNs, MSN-PLH and MSN-PLH-TAM; where the colour of the particles changed from white to red upon loading (Figure 3 a shows the colour change for the MSN-PLH-TAM system). In Figure 3b, the FT-IR spectra of DOX@MSN-PLH-TAM demonstrated the same peaks as the unloaded MSN-PLH-TAM which was in accordance with our previously published study [16]. No additional signal was obtained; this suggests that the loaded DOX content might be just too low to be detected by FT-IR. This was also consistent for the MSN-PLH vs. DOX@MSN-PLH system (see Figure S2 in Supplementary Material).

DOX@MSN-PLH achieved an EE of 12.2\% while DOX@MSN-PLH-TAM achieved a similar EE of $12.1 \%$. The EE of these functionalized systems was slightly greater than that of DOX@MSN. This may be due to enhanced interactions between the DOX molecules and the hydrophobic PLH molecules due to $\pi-\pi$ stacking [36], leading to an increased amount of DOX becoming encapsulated. Interestingly, this increased trend was not observed with the DC of both functionalized systems. DOX@MSN-PLH and MSN-PLH-TAM achieved a DC of $\approx 7 \%$, which is very similar to that of the native MSNs. 
(a)

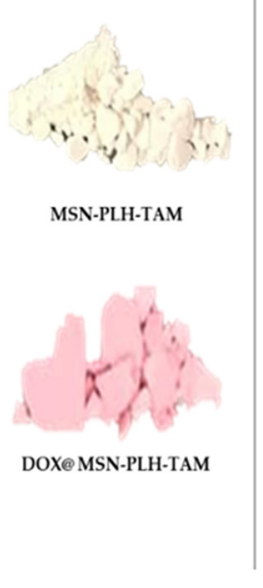

(b)

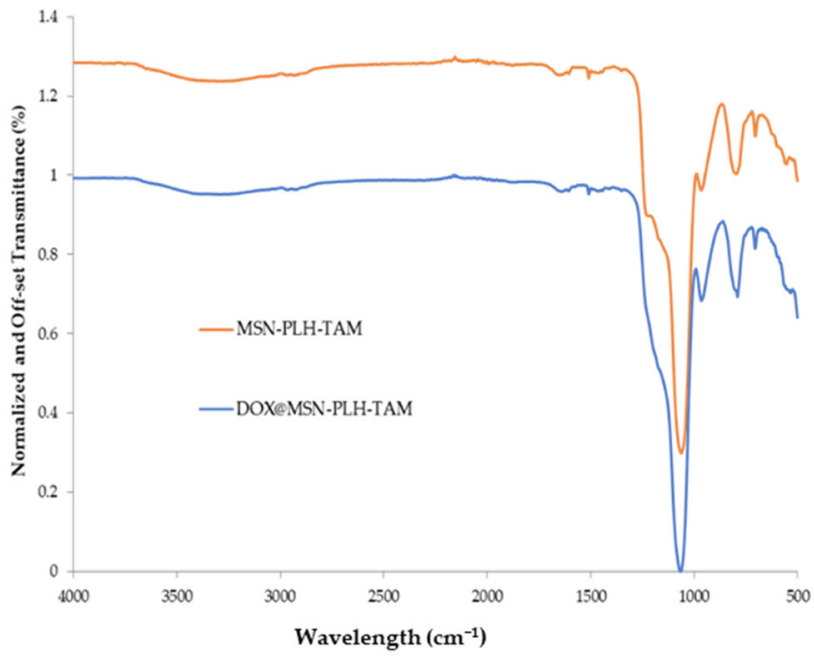

Figure 3. (a) Photos of MSN-PLH-TAM vs. DOX@MSN-PLH-TAM, highlighting the colour change upon DOX loading. (b) FT-IR spectra of two MSN-based systems. No additional signals were observed following the loading of DOX into the MSN-PLH-TAM.

\subsection{Drug Release}

The next step in the development of our nanosystem was to characterize the release of DOX. This was done using a diffusion bag technique, where each MSN-based- system was loaded into a dialysis bag and the release media collected at varying time points. For this experiment a positive control was employed, where pure DOX was loaded into the dialysis bag and the releasing media consisted of either the $\mathrm{pH} 7.4$ or $\mathrm{pH} 5$ buffer systems. The media collected was then analysed by HPLC, in which the characteristic peak of pure DOX was obtained at $4.3 \mathrm{~min}$, while the peak eluted before three minutes were the solvent (buffers) peak as per previous literature [33].

The first systems to be evaluated were those synthesized by the pre-surface grafting loading method (Figure 2a). When the DOX@MSNs from the pre-grafting loading method were evaluated for release, DOX was detected in both the release mediums of $\mathrm{pH} 7.4$ and 5 (Figure 4). This indicated that encapsulated DOX was liberated into the releasing media. In addition to this, the peak of DOX from DOX@MSN at pH 7.4 has a lower AUC compared to the peak of DOX from DOX@MSN at pH 5, indicating a higher release of DOX from native MSNs under acidic conditions. This is as expected as DOX is known to be more soluble at acidic $\mathrm{pH}[31,32]$.

Unfortunately, when the release of the functionalized particles DOX@MSN-PLH-TAM (synthesized by pre-surface grafting method, Figure 2a) was evaluated using the same release procedure, no DOX was detected in the release media. It was hypothesized that there was insufficient amount of DOX left within the mesopores after multiple surface grafting steps and that what remained was well below the detection limit of the instrument. This is consistent with the similar FT-IR spectrum of the DOX loaded systems vs. unloaded systems, implying a low loading of DOX (Figure 3; Figures S1 and S2).

To investigate the in vitro release pattern of DOX@MSN-PLH and DOX@MSN-PLHTAM (synthesized by post-surface grafting loading method, Figure $2 b$ ), the release of DOX from native MSNs was used as a control for comparison. The release kinetic of DOX@MSNs, DOX@MSN-PLH and DOX@MSN-PLH-TAM were demonstrated in Figure 5 below. 

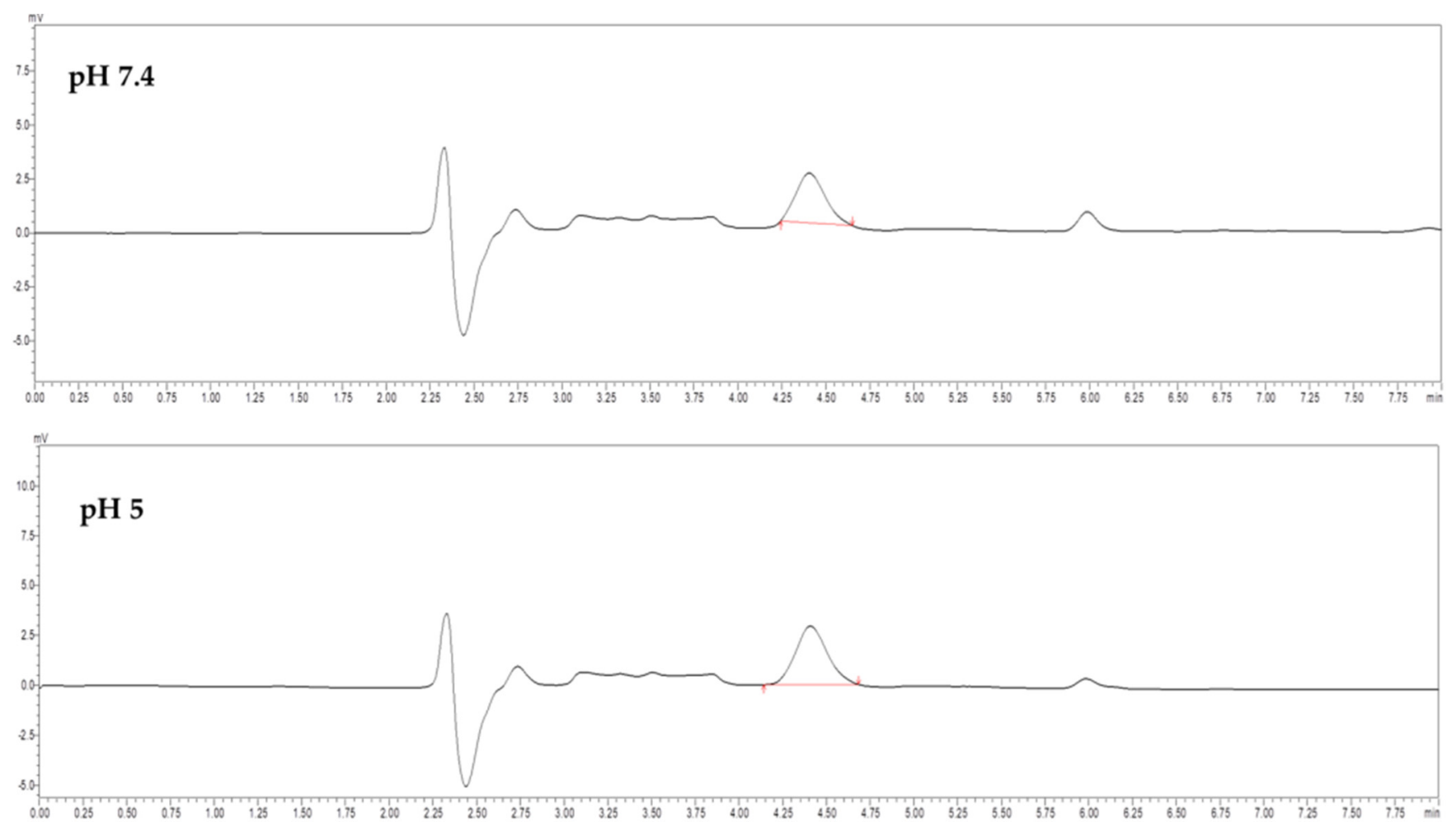

Figure 4. Chromatography of demonstrating the in vitro release kinetics of DOX@MSNs at pH 7.4 and 5.

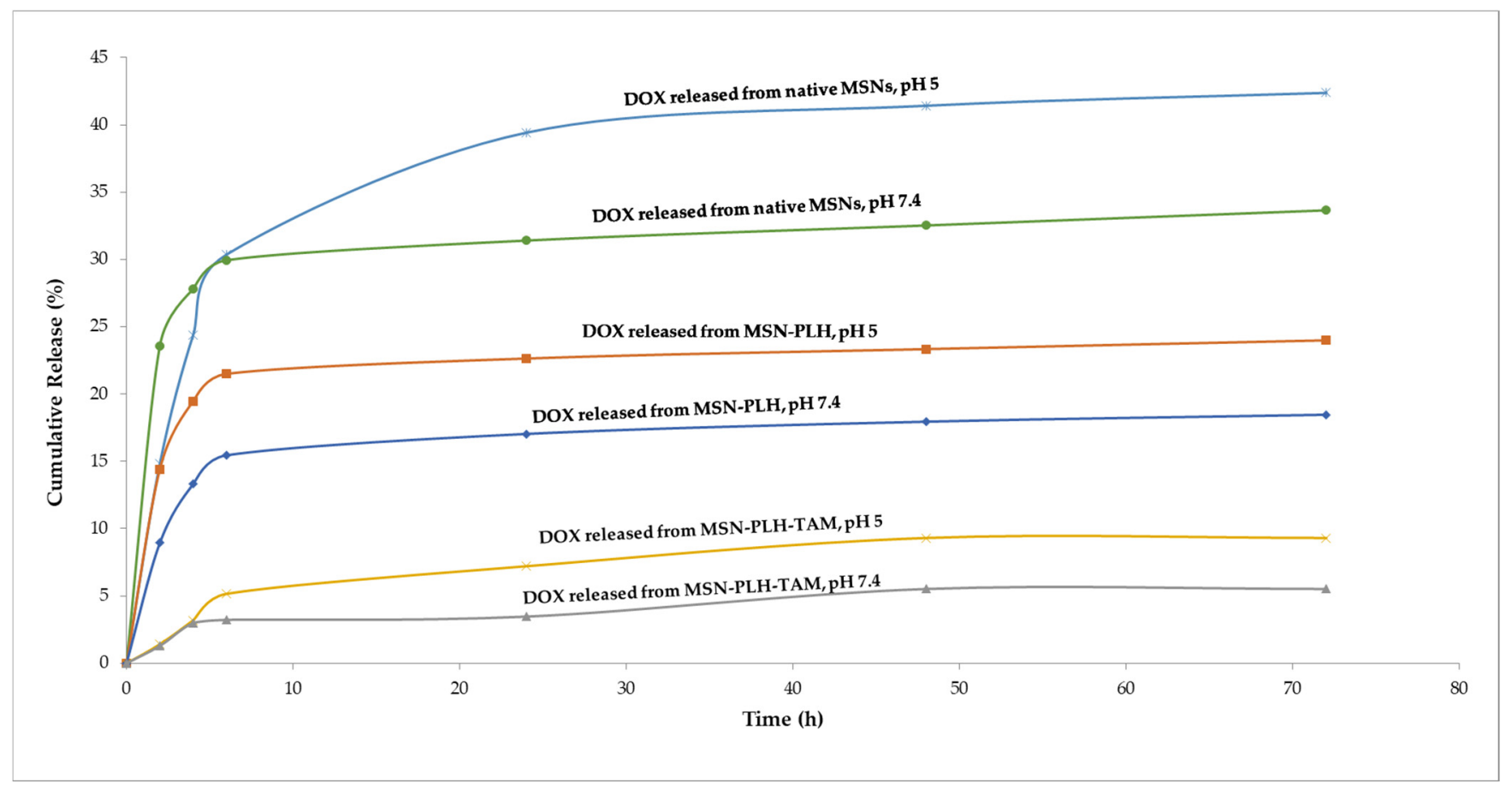

Figure 5. In vitro cumulative release profile of DOX@MSN, DOX@MSN-PLH and DOX@MSN-PLH-TAM over 72 h.

More DOX was released at acidic $\mathrm{pH}$ in all three systems; native MSNs, MSN-PLH and MSN-PLH-TAM. This reaffirmed the knowledge that DOX molecules are more soluble in acidic environments [31,32]. For native MSNs, 8.7\% more DOX was released at $\mathrm{pH} 5$ than at $\mathrm{pH}$ 7.4. It was observed that the release of DOX@MSNs reached a plateau, with an overall release after $72 \mathrm{~h}$ of $33.6 \%$ for DOX at $\mathrm{pH} 7.4$; and $42.3 \%$ at $\mathrm{pH} 5$. However, the total amount of DOX released from the native MSNs was less than typically expected from this 
type of material, where a release of over $80 \%$ after $24 \mathrm{~h}$ is reported $[30,37]$. This incomplete release from native MSN may be attributed to absorption of DOX molecules inside the deep channels of our materials, requiring the need for releasing media to penetrate inside and solubilize the drug, hence, prolonging the releasing time.

For functionalized MSNs, it was observed that the cumulative amount of DOX released from MSN-PLH and MSN-PLH-TAM was less than that of native MSNs. The maximal cumulated release of DOX molecules from DOX@MSN-PLH after $72 \mathrm{~h}$ was $\approx 18 \%$ at $\mathrm{pH} 7.4$ and $\approx 23 \%$ at $\mathrm{pH} 5$, respectively. Whereas approximately $5 \%$ of DOX was released from DOX@MSN-PLH-TAM at pH 7.4 and 9\% released at pH 5 over 72 h (Figure 5). Due to the complexity of the synthesis for functionalized systems, the release experiments of DOX@MSN-PLH and DOX@MSN-PLH-TAM (synthesized by post-surface grafting approach, Figure $2 \mathrm{~b}$ ) were only conducted once for each sample. However, the pilot study still confirmed a decrease in DOX release from DOX@MSN-PLH and DOX@MSN-PLHTAM system, compared to that of native DOX@MSNs. This implied a potential pore blocking effect due to the addition of surface functionalities. Additionally, this may be due to the $(-\mathrm{OH})$ groups of encapsulated DOX molecules forming some additional hydrogen bonds with amine groups (from the lysine moiety) of the PLH molecules and/or with the oxygen atoms of TAM molecules.

We observed a $\mathrm{pH}$ sensitive release behaviour of DOX in our novel nanostructure MSN-PLH-TAM, with more DOX released at acidic $\mathrm{pH}$ compared to that of biological $\mathrm{pH}$. However, the release of encapsulated DOX from MSN-PLH-TAM decreased at both pHs, compared to that of MSN-PLH. This may suggest that obtained delayed release was a result of the pore-blocking effect caused by the increased surface moieties, following the conjugation of TAM-TEG building blocks onto MSN-PLH. In addition to this, it is possible that some hydrophobic stacking between TAM and PLH molecules may have occurred on the surface of MSN-PLH-TAM, contributing to its overall lower amount of DOX released over time.

\section{Conclusions}

DOX was successfully encapsulated into three MSN-based systems, namely MSN, MSN-PLH and MSN-PLH-TAM, using a post grafting loading method. The release kinetics in all three systems demonstrated a $\mathrm{pH}$-sensitive pattern, with more DOX released at acidic $\mathrm{pH}$. Following the attachment of TAM molecules into the MSN-PLH, the pH-effect was still retained. Despite the lower amount of DOX released from our functionalized system as compared with native MSNs, our developed functionalized MSNs were able to demonstrate an effect of $\mathrm{pH}$ on the release kinetics. Even though obtained data demonstrated expected $\mathrm{pH}$ - sensitive patterns of the functionalized systems, more in vitro pharmacodynamics investigations need to be performed, to confirm if this effect is sufficient to off-set the dose and drug-associated toxicities of DOX on healthy cells, while still maintaining its efficacy against tumours. Our future work will improve the release of DOX, as well as investigate the targeting capacity of our systems against breast cancer with cellular uptake studies.

Supplementary Materials: The following are available online at https: / www.mdpi.com/article / 10.3390/app11136121/s1, Figure S1: FT-IR spectrum of DOX@MSNs vs unloaded native MSNs, Figure S2: FT-IR spectrum of DOX@MSN-PLH vs unloaded MSN-PLH.

Author Contributions: Conceptualization, C.M.D., S.E.P. and S.G.; methodology, C.M.D., M.J.S., S.E.P., Y.S. and S.G.; validation, C.M.D.; formal analysis, C.M.D.; investigation, C.M.D. and M.J.S.; resources, S.E.P., S.G. and Y.S.; data curation, C.M.D.; writing-original draft preparation, C.M.D.; writing-review and editing.; S.G., S.E.P., M.J.S. and Y.S.; visualization, C.M.D.; supervision, S.E.P., Y.S. and S.G.; project ad-ministration, Y.S., S.E.P. and S.G.; funding acquisition, S.G. and S.E.P. All authors have read and agreed to the published version of the manuscript.

Funding: This research received no external funding.

Institutional Review Board Statement: Not applicable. 
Informed Consent Statement: Not applicable.

Acknowledgments: The authors would like to acknowledge all the members of the Pharmaceutical Innovation and Development Group (PIDG-UniSA) and Bioinorganic Synthesis and Imaging Group (BSIG-UniSA) for their support throughout this work.

Conflicts of Interest: The authors declare no conflict of interest.

\section{References}

1. Bleeker, E.A.; de Jong, W.H.; Geertsma, R.E.; Groenewold, M.; Heugens, E.H.; Koers-Jacquemijns, M.; van de Meent, D.; Popma, J.R.; Rietveld, A.G.; Wijnhoven, S.W.; et al. Considerations on the EU definition of a nanomaterial: Science to support policy making. Regul. Toxicol. Pharmacol. 2013, 65, 119-125. [CrossRef]

2. Day, C.M.; Hickey, S.M.; Song, Y.; Plush, S.E.; Garg, S. Novel Tamoxifen Nanoformulations for Improving Breast Cancer Treatment: Old Wine in New Bottles. Molecules 2020, 25, 1182. [CrossRef]

3. Day, C.M.; Barclay, T.G.; Song, Y.; Garg, S. Swelling-controlled Drug Delivery Systems. In Biomaterials Science Series; The Royal Society of Chemistry: London, UK, 2018; Volume 2018, pp. 232-264.

4. Jena, S.K.; Sangamwar, A.T. Polymeric micelles: A promising tool for tamoxifen delivery in cancer? Ther. Deliv. 2017, 8, 109-111. [CrossRef]

5. Xuan, Q.J.; Wang, J.X.; Nanding, A.; Wang, Z.P.; Liu, H.; Lian, X.; Zhang, Q.-Y. Tumor-Associated Macrophages are Correlated with Tamoxifen Resistance in the Postmenopausal Breast Cancer Patients. Pathol. Oncol. Res. 2014, 20, 619-624. [CrossRef]

6. Singh, R.; Lillard, J.W., Jr. Nanoparticle-based targeted drug delivery. Exp. Mol. Pathol. 2009, 86, 215-223. [CrossRef]

7. Ventola, C.L. Progress in Nanomedicine: Approved and Investigational Nanodrugs. Pharm. Ther. 2017, 42, 742-755.

8. Mitchell, M.J.; Billingsley, M.M.; Haley, R.M.; Wechsler, M.E.; Peppas, N.A.; Langer, R. Engineering precision nanoparticles for drug delivery. Nat. Rev. Drug Discov. 2021, 20, 101-124. [CrossRef]

9. Farjadian, F.; Ghasemi, A.; Gohari, O.; Roointan, A.; Karimi, M.; Hamblin, M.R. Nanopharmaceuticals and nanomedicines currently on the market: Challenges and opportunities. Nanomedicine 2019, 14, 93-126. [CrossRef] [PubMed]

10. Narayan, R.; Nayak, U.Y.; Raichur, A.M.; Garg, S. Mesoporous Silica Nanoparticles: A Comprehensive Review on Synthesis and Recent Advances. Pharmaceutics 2018, 10, 118. [CrossRef] [PubMed]

11. Day, C.M.; Sweetman, M.J.; Garg, S. Mesoporous silica nanoparticles for drug combination delivery in cancer therapy: Background, current insights, and future perspectives. In Nanocarriers for the Delivery of Combination Drugs; Elsevier: Amsterdam, The Netherlands, 2021; Volume 2021, pp. 119-162.

12. Keshavarz, M.; Ahmad, N. Characterization and Modification of Mesoporous Silica Nanoparticles Prepared by Sol-Gel. J. Nanoparticles 2013, 2013, 102823. [CrossRef]

13. Keshavarz, M.; Tan, B.; Venkatakrishnan, K. Cell Selective Apoptosis Induced by Polymorphic Alteration of Self-Assembled Silica Nanowebs. ACS Appl. Mater. Interfaces 2017, 9, 6292-6305. [CrossRef]

14. Zhao, Y.; Sun, X.; Zhang, G.; Trewyn, B.G.; Slowing, I.I.; Lin, V.S.Y. Interaction of Mesoporous Silica Nanoparticles with Human Red Blood Cell Membranes: Size and Surface Effects. ACS Nano 2011, 5, 1366-1375. [CrossRef]

15. Slowing, I.I.; Wu, C.W.; Vivero-Escoto, J.L.; Lin, V.S. Mesoporous silica nanoparticles for reducing hemolytic activity towards mammalian red blood cells. Small 2009, 5, 57-62. [CrossRef] [PubMed]

16. Day, C.M.; Sweetman, M.J.; Hickey, S.M.; Song, Y.; Liu, Y.; Zhang, N.; Plush, S.E.; Garg, S. Concept Design, Development and Preliminary Physical and Chemical Characterization of Tamoxifen-Guided-Mesoporous Silica Nanoparticles. Molecules 2021, 26, 219. [CrossRef]

17. Shen, S.; Wu, Y.; Liu, Y.; Wu, D. High drug-loading nanomedicines: Progress, current status, and prospects. Int. J. Nanomed. 2017, 12, 4085-4109. [CrossRef] [PubMed]

18. Lao, J.; Madani, J.; Puértolas, T.; Alvarez, M.; Hernández, A.; Pazo-Cid, R.; Artal, A.; Antón Torres, A. Liposomal Doxorubicin in the treatment of breast cancer patients: A review. J. Drug. Deliv. 2013, 2013, 456409. [CrossRef]

19. McGowan, J.V.; Chung, R.; Maulik, A.; Piotrowska, I.; Walker, J.M.; Yellon, D.M. Anthracycline Chemotherapy and Cardiotoxicity. Cardiovasc. Drugs Ther. 2017, 31, 63-75. [CrossRef]

20. Zhao, M.; Ding, X.F.; Shen, J.Y.; Zhang, X.P.; Ding, X.W.; Xu, B. Use of liposomal doxorubicin for adjuvant chemotherapy of breast cancer in clinical practice. J. Zhejiang Univ. Sci. B 2017, 18, 15-26. [CrossRef]

21. Jafari, S.; Derakhshankhah, H.; Alaei, L.; Fattahi, A.; Varnamkhasti, B.S.; Saboury, A.A. Mesoporous silica nanoparticles for therapeutic/diagnostic applications. Biomed. Pharmacother. 2019, 109, 1100-1111. [CrossRef] [PubMed]

22. Bansal, K.K.; Mishra, D.K.; Rosling, A.; Rosenholm, J.M. Therapeutic Potential of Polymer-Coated Mesoporous Silica Nanoparticles. Appl. Sci. 2020, 10, 289. [CrossRef]

23. Amolegbe, S.A.; Hirano, Y.; Adebayo, J.O.; Ademowo, O.G.; Balogun, E.A.; Obaleye, J.A.; Krettli, A.U.; Yu, C.; Hayami, S. Mesoporous silica nanocarriers encapsulated antimalarials with high therapeutic performance. Sci. Rep. 2018, 8, 3078. [CrossRef]

24. Wang, H.; Li, X.; Ma, Z.; Wang, D.; Wang, L.; Zhan, J.; She, L.; Yang, F. Hydrophilic mesoporous carbon nanospheres with high drug-loading efficiency for doxorubicin delivery and cancer therapy. Int. J. Nanomed. 2016, 11, 1793-1806. 
25. Ji, F.; Sun, H.; Qin, Z.; Zhang, E.; Cui, J.; Wang, J.; Li, S.; Yao, F. Engineering Polyzwitterion and Polydopamine Decorated Doxorubicin-Loaded Mesoporous Silica Nanoparticles as a pH-Sensitive Drug Delivery. Polymers 2018, 10, 326. [CrossRef] [PubMed]

26. Chen, Y.; Ai, K.; Liu, J.; Sun, G.; Yin, Q.; Lu, L. Multifunctional envelope-type mesoporous silica nanoparticles for pH-responsive drug delivery and magnetic resonance imaging. Biomaterials 2015, 60, 111-120. [CrossRef] [PubMed]

27. Narayan, R.; Gadag, S.; Cheruku, S.P.; Raichur, A.M.; Day, C.M.; Garg, S.; Manandhar, S.; Sreedhar, K.; Pai, R.; Suresh, A.; et al Chitosan-glucuronic acid conjugate coated mesoporous silica nanoparticles: A smart pH-responsive and receptor-targeted system for colorectal cancer therapy. Carbohydr. Polym. 2021, 261, 117893. [CrossRef]

28. Varache, M.; Bezverkhyy, I.; Weber, G.; Saviot, L.; Chassagnon, R.; Baras, F.; Bouyer, F. Loading of Cisplatin into Mesoporous Silica Nanoparticles: Effect of Surface Functionalization. Langmuir 2019, 35, 8984-8995. [CrossRef]

29. Zhou, S.; Wu, D.; Yin, X.; Jin, X.; Zhang, X.; Zheng, S.; Wang, C.; Liu, Y. Intracellular pH-responsive and rituxi-mab-conjugated mesoporous silica nanoparticles for targeted drug delivery to lymphoma B cells. J. Exp. Clin. Cancer. Res. 2017, 36, 24. [CrossRef] [PubMed]

30. Subedi, R.K.; Kang, K.W.; Choi, H.K. Preparation and characterization of solid lipid nanoparticles loaded with doxorubicin. Eur. J. Pharm. Sci. 2009, 37, 508-513. [CrossRef] [PubMed]

31. Janssen, M.J.H.; Crommelin, D.J.A.; Storm, G.; Hulshoff, A. Doxorubicin decomposition on storage. Effect of pH, type of buffer and liposome encapsulation. Int. J. Pharm. 1985, 23, 1-11. [CrossRef]

32. Hakeem, A.; Zahid, F.; Zhan, G.; Yi, P.; Yang, H.; Gan, L.; Yang, X. Polyaspartic acid-anchored mesoporous silica nanoparticles for pH-responsive doxorubicin release. Int. J. Nanomed. 2018, 13, 1029-1040. [CrossRef]

33. Wang, L.; Song, Y.; Parikh, A.; Joyce, P.; Chung, R.; Liu, L.; Afinjuomo, F.; Hayball, J.D.; Petrovsky, N.; Barclay, T.G.; et al. Doxorubicin-Loaded Delta Inulin Conjugates for Controlled and Targeted Drug Delivery: Development, Characterization, and In Vitro Evaluation. Pharmaceutics 2019, 11, 581. [CrossRef]

34. Sun, J.G.; Jiang, Q.; Zhang, X.P.; Shan, K.; Liu, B.; Zhao, C.; Yan, B. Mesoporous silica nanoparticles as a delivery system for improving antiangiogenic therapy. Int. J. Nanomed. 2019, 14, 1489-1501. [CrossRef]

35. Shen, J.; He, Q.; Gao, Y.; Shi, J.; Li, Y. Mesoporous silica nanoparticles loading doxorubicin reverse multidrug resistance: Performance and mechanism. Nanoscale 2011, 3, 4314-4322. [CrossRef] [PubMed]

36. Bilalis, P.; Tziveleka, L.A.; Varlasa, S.; Iatrou, H. pH-Sensitive nanogates based on poly(l-histidine) for controlled drug release from mesoporous silica nanoparticles. Polym. Chem. 2016, 7, 1475-1485. [CrossRef]

37. Wu, X.; Wang, Z.; Zhu, D.; Zong, S.; Yang, L.; Zhong, Y.; Cui, Y. pH and Thermo Dual-Stimuli-Responsive Drug Carrier Based on Mesoporous Silica Nanoparticles Encapsulated in a Copolymer-Lipid Bilayer. ACS Appl. Mater. Interfaces 2013, 5, 10895-10903. [CrossRef] [PubMed]

38. Duo, Y.; Li, Y.; Chen, C.; Liu, B.; Wang, X.; Zeng, X.; Chen, H. DOX-loaded pH-sensitive mesoporous silica nanoparticles coated with PDA and PEG induce pro-death autophagy in breast cancer. RSC Adv. 2017, 7, 39641-39650. [CrossRef] 